\title{
Effects of etizolam and ethyl loflazepate on the P300 event-related potential in healthy subjects
}

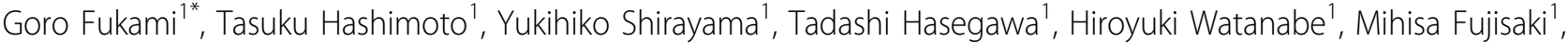 \\ Kenji Hashimoto ${ }^{2}$, Masaomi lyo ${ }^{1}$
}

\begin{abstract}
Background: Benzodiazepines carry the risk of inducing cognitive impairments, which may go unnoticed while profoundly disturbing social activity. Furthermore, these impairments are partly associated with the elimination halflife (EH) of the substance from the body. The object of the present study was to examine the effects of etizolam and ethyl loflazepate, with EHs of $6 \mathrm{~h}$ and $122 \mathrm{~h}$, respectively, on information processing in healthy subjects.

Methods: Healthy people were administered etizolam and ethyl loflazepate acutely and subchronically (14 days). The auditory P300 event-related potential and the neuropsychological batteries described below were employed to assess the effects of drugs on cognition. The P300 event-related potential was recorded before and after drug treatments. The digit symbol test, trail making test, digit span test and verbal paired associates test were administered to examine mental slowing and memory functioning.

Results: Acute administration of drugs caused prolongation in P300 latency and reduction in P300 amplitude. Etizolam caused a statistically significant prolongation in P300 latency compared to ethyl loflazepate. Furthermore, subchronic administration of etizolam, but not ethyl loflazepate, still caused a weak prolongation in P300 latency. In contrast, neuropsychological tests showed no difference.
\end{abstract}

Conclusions: The results indicate that acute administration of ethyl loflazepate induces less effect on P300 latency than etizolam.

\section{Background}

Benzodiazepines have anxiolytic, sedative, anticonvulsant and myorelaxant properties, and have been widely prescribed in various clinical settings. These compounds, however, also induce adverse effects such as oversedation, cognitive impairment, motor impairment and withdrawal. These adverse effects may be partly associated with the elimination half-life $(\mathrm{EH})$ of the compounds from the body; that is, long-term use of the compounds with a short elimination rate may induce withdrawal syndromes, whereas accumulation-related effects of a long elimination rate may include oversedation, cognitive dysfunction and motor impairment [1-4].

It has been observed previously that cognitive impairment induced by benzodiazepines may go unnoticed while profoundly disturbing social activity [5]. Therefore,

\footnotetext{
* Correspondence: fukami@faculty.chiba-u.jp

'Department of Psychiatry, Chiba University Graduate School of Medicine,

Chiba, Japan

Full list of author information is available at the end of the article
}

it is clinically very important to take note of the cognitive effects of benzodiazepines. In order to assess the effects of benzodiazepines on cognition, the event-related potential (ERP), P300, may be useful [6], as well as neuropsychological tests. The P300 components of ERP are elicited by an auditory oddball paradigm in which a subject detects infrequent task-relevant stimuli randomly presented among frequent stimuli. P300 reflects stimulus context and stimulus meaning [7]. P300 components are associated with cognitive processes such as attention, memory, orientation and evaluation. Relationships between P300 and neuropsychological function have been reported [8-14]. Benzodiazepine anxiolytic drugs, as well as benzodiazepine hypnotic drugs, have been reported to induce reductions in P300 amplitude and prolongation in P300 latency [6,15-20].

As far as is known, however, there are no reports on the effects of chronic or subchronic administration of benzodiazepines on cognition and P300 from the viewpoint of elimination rates. Here, we studied the effects 
of anxiolytic benzodiazepines on neuropsychological functions and P300 components of auditory ERP under acute and subchronic administration of ethyl loflazepate and etizolam. Ethyl loflazepate is a potent, non-sedative, anxiolytic drug with a long EH of $122 \mathrm{~h}$ [21], whereas etizolam is characteristic of a potent antianxiety and sedative drug with a short EH of $6 \mathrm{~h}$ [22]. Therefore, etizolam is often used as a sleep inducer. However, it is well known that benzodiazepine drugs including etizolam and ethyl loflazepate have the effects of reducing the deep sleep stage 3, resulting in loss of good sleep. The aim of the present study is to examine whether sedative or anxiolytic actions of benzodiazepines have some effects on ERP and neuropsychological tests.

\section{Methods}

\section{Study design and subjects}

All subjects had normal acoustic function and were right handed. The ethics committee of Chiba University Graduate School of Medicine approved the experiments. Subjects were free from treatment for past psychiatric illness. Written informed consent was obtained after the procedure had been fully explained.

In the acute experiment, 10 healthy men $(n=5)$ and women $(\mathrm{n}=5)$ ranging in age from 16 to 38 (average age 28.6 (SD 6.5)) participated in the study. First, all subjects were measured for the P300 components of ERP and received neuropsychological tests. Then they took etizolam ( 1 or $2 \mathrm{mg}$, orally). Then, $2 \mathrm{~h}$ later, the same ERP and neuropsychological tests were performed, since the blood concentration of the drugs reaches a maximum 1-2 $\mathrm{h}$ after consumption. After a 2-week washout period, the same experimental procedures were repeated, but subjects took ethyl loflazepate ( 1 or $2 \mathrm{mg}$, orally).

In the subchronic experiment, 17 healthy men $(\mathrm{n}=8)$ and women $(\mathrm{n}=9)$ ranging in age from 22 to 34 (average age 27.4 (SD 4.1)) participated in the study. The 17 subjects were divided into 2 groups: the first group was given etizolam ( $1 \mathrm{mg}$, orally, for 14 days), and the second given ethyl loflazepate (1 mg, orally for 14 days). Subjects were asked to take drugs in the evening every day, and performed ERP recording and neuropsychological tests 14-20 h after taking the last drug. Subjects performed ERP recording and neuropsychological tests twice before and after subchronic treatment with etizolam or ethyl loflazepate.

Doses examined in the present study were chosen based on the equivalent conversion table for anxiolytic drugs ( $5 \mathrm{mg}$ of diazepam, $1.5 \mathrm{mg}$ of etizolam, and 1.67 mg of ethyl loflazepate) [23,24].

\section{ERP procedure}

Electroencephalogram electrodes were attached at $\mathrm{Fz}, \mathrm{Cz}$ and $\mathrm{Pz}$ according to the international 10-20 system.
Earlobe electrodes were linked for reference. Electrooculography was also recorded from vertical and lateral derivations to check ocular artefacts. Subjects sat on a semi-reclined chair in a sound-attenuated and electrically shielded room during recordings. Subjects were instructed to press a button as quickly as possible upon hearing the infrequent high-pitched tones. Event-related potentials were recorded under an oddball paradigm. The stimuli consisted of a $1,000 \mathrm{~Hz}$ tone burst (frequent non-target stimulus) and a 2,000 $\mathrm{Hz}$ tone burst (rare target stimulus). In each paradigm, 200 stimuli were presented through bilateral earphones by using a Neuropack 10 (MEB-2200, Nihon Kohden, Tokyo, Japan). The ratio of the rare versus frequent stimuli was 0.25 . Stimuli were presented in a random order, the duration of each stimulus being $120 \mathrm{~ms}$, with rise and fall times of $10 \mathrm{~ms}$. The intensity was $40 \mathrm{~dB}$ for all stimuli. The interstimulus interval was $1.5 \mathrm{~s}$.

\section{Neuropsychological tests}

The trail making test consists of two parts [25]. In part A, subjects are asked to draw lines connecting 25 consecutively numbered circles on a worksheet. In part B, they draw lines connecting 25 consecutively numbered and lettered circles, alternating between the sequences (for example, 1-A-2-B-3 and so on). Part A examines psychomotor speed and attention. Part $B$ examines set alternation or divided attention.

The digit symbol modalities test is a measure of switching attention [26]. Subjects are asked to identify nine different symbols corresponding to the numbers 1 through 9, and write the correct number under the corresponding symbol. Thus, visual shifting and pairing of specific digits is directed, with a set of prespecified symbols.

The forward digit span test is a measure of simple attention, immediate memory and attentional control processing. In contrast, backward digit span is not only a test of attentional control processing but also working memory test.

The verbal paired associates test from the Wechsler Memory Scale-Revised (WMS-R) is a cued recall test of verbal memory [27]. Subjects learned a list of eight verbal paired associates. Then, either immediately or after a delay, the examiner says one word of each pair and the subjects recall the other word. Three sets of immediate memory testing and one set of delayed recall testing were administered.

\section{Statistical analysis}

Two-way repeated measures analysis of variance (ANOVA) was performed to assess the overall differences between variables. Where a significant interaction in the within-subject variables was found, subsequent one-way 
ANOVA was carried out among more than three groups by a post hoc comparison using Fisher's protected least significant difference test. For comparison of the mean values between the two groups, statistical evaluation was performed using the two-tailed Student's t test. The significance level was set at $P<0.05$.

\section{Results}

\section{Effects of acute treatments with etizolam and ethyl} loflazepate on P300

For acute drug treatment on the P300, two-way repeated ANOVA indicated significant effects of treatment $(\mathrm{Fz}, \mathrm{F}$ $(1,16)=49.397, P<0.0001 ; \mathrm{Cz}, \mathrm{F}(1,16)=59.022, P<$ 0.0001; Pz, F $(1,16)=45.623, P<0.0001)$, but not effects of group, on latency, with a significant interaction $(\mathrm{Fz}$, treatment $\times$ group, $\mathrm{F}(3,16)=3.846, P=0.0301 ; \mathrm{Cz}$, treatment $\times$ group, $\mathrm{F}(3,16)=3.436, P=0.0423 ; \mathrm{Pz}$, treatment $\times$ group, $\mathrm{F}(3,16)=3.278, P=0.0483$ ) (Figure $1 \mathrm{a}-\mathrm{c})$. The subsequent one-way ANOVA on the changes of P300 latency indicated significant differences $(\mathrm{Fz}, \mathrm{F}$ $(3,16)=3.431, P=0.0425 ; \mathrm{Cz}, \mathrm{F}(3,16)=3.436, P=$ 0.0423; Pz, F $(3,16)=3.387, P=0.0441)$, and the post hoc comparison using Fisher's protected least significant difference test indicated the following: ethyl loflazepate $1 \mathrm{mg}$ has less effects than etizolam $1 \mathrm{mg}$ and etizolam 2 $\mathrm{mg}$ in $\mathrm{Fz}$ (Figure 1g), $\mathrm{Cz}$ (Figure $1 \mathrm{~h}$ ), and $\mathrm{Pz}$ (Figure 1i). For amplitude, two-way repeated ANOVA indicated significant effects of treatment $(\mathrm{Cz}, \mathrm{F}(1,16)=7.967, P=$ $0.0123 ; \mathrm{Pz}, \mathrm{F}(1,16)=8.807, P=0.0091$; but see $\mathrm{Fz}, \mathrm{F}$ $(1,16)=4.032, P=0.0618)$, but not effects of group, without a significant interaction (Figure 1d-f). This seems to reflect that benzodiazepine reduced P300 amplitude. Although the subsequent one-way ANOVA on the changes of P300 amplitude revealed no significant difference among drug groups (Figure $1 j-1$ ), the magnitude of changes showed that etizolam $(2 \mathrm{mg})$ produced a trend in reduction of amplitude.

\section{Effects of subchronic treatments with etizolam and ethyl loflazepate on P300}

For subchronic drug treatment on the P300, two-way repeated ANOVA indicated significant effects of treatment region specifically $(\mathrm{Fz}, \mathrm{F}(1,15)=7.734, P=0.0140$; but see $\mathrm{Cz}, \mathrm{F}(1,15)=2.391, P=0.1491 ; \mathrm{Pz}, \mathrm{F}(1,15)=$

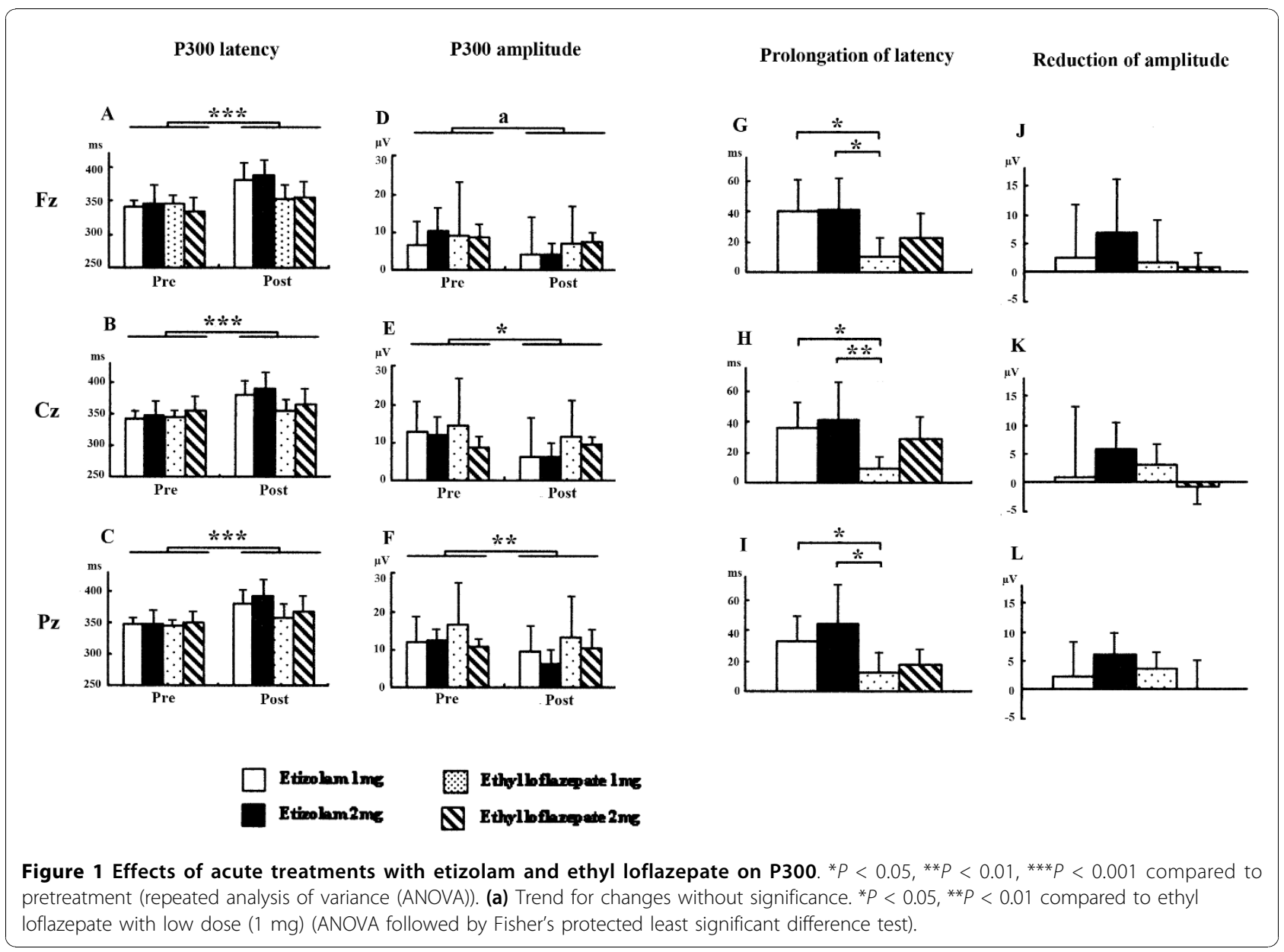


0.954, $P=0.3443$ ), but not effects of group, on latency, without a significant interaction (Figure $2 \mathrm{a}-\mathrm{c}$ ). The subsequent Student $t$ test on the changes of P300 latency revealed no significant difference between two drugs (Figure 2g-i). With regard to amplitude, two-way repeated ANOVA indicated no significant effects of treatment without a significant interaction (Figure 2d-f, j-l).

\section{Effects of treatments with etizolam and ethyl loflazepate on neuropsychological tests}

For acute effects of drug treatment on neuropsychological tests, two-way repeated ANOVA showed significant practice effects of repeated testing, but not effects of drug group, on test scoring without a significant interaction in some tests including trail making $A(F(1,16)=$ 7.399, $P=0.0151$, Figure 3a), trail making $B(F(1,16)=$ $8.409, P=0.0104$, Figure $3 \mathrm{~b}$ ), digit span forward (F $(1,16)=8.696, P=0.0094$, Figure $3 \mathrm{c})$, verbal paired associates immediate memory $(\mathrm{F}(1,16)=6.485, P=$ 0.0215 , Figure $3 \mathrm{e})$ and digit symbol $(\mathrm{F}(1,16)=24.209, P$ $=0.0002$, Figure $3 \mathrm{~g}$ ), and no significant effects of repeated testing and drugs on test scoring without a significant interaction in other tests such as digit span backward (Figure 3d) and verbal paired associates delayed recall (Figure $3 \mathrm{f}$ ).
For subchronic effects of drug treatment on neuropsychological tests, two-way repeated ANOVA indicated significant practice effects of repeated testing, but not effects of drug group, on test scoring without a significant interaction, in some tests including trail making test $\mathrm{A}(\mathrm{F}(1,15)=12.472, P=0.0030$, Figure $3 \mathrm{~h})$, trail making test $\mathrm{B}(\mathrm{F}(1,15)=5.426, P=0.0342$, Figure $3 \mathrm{i})$, digit span forward $(\mathrm{F}(1,15)=7.092, P=0.0177$, Figure $3 \mathrm{j})$, verbal paired associates immediate memory $(\mathrm{F}(1,15)$ $=16.449, P=0.0010$, Figure 31), verbal paired associates delayed recall $(\mathrm{F}(1,15)=5.773, P=0.0297$, Figure $3 \mathrm{~m})$ and digit symbol $(\mathrm{F}(1,15)=6.075, P=0.0236$, Figure $3 n$ ), and no significant effects of repeated testing and drug group on test scoring without a significant interaction in digit span backward test (Figure 3k).

\section{Discussion}

Our results show acute drug treatment induced prolongation in P300 latency. This is consistent with previous studies demonstrating that benzodiazepines such as alprazolam, lorazepam, clonazepam and triazolam induce prolongation in P300 latency $[6,16,18,19]$. However, subsequent ANOVA revealed that etizolam (1 and $2 \mathrm{mg}$ ) induced significant prolongation in P300 latency compared to ethyl loflazepate $(1 \mathrm{mg})$. The difference

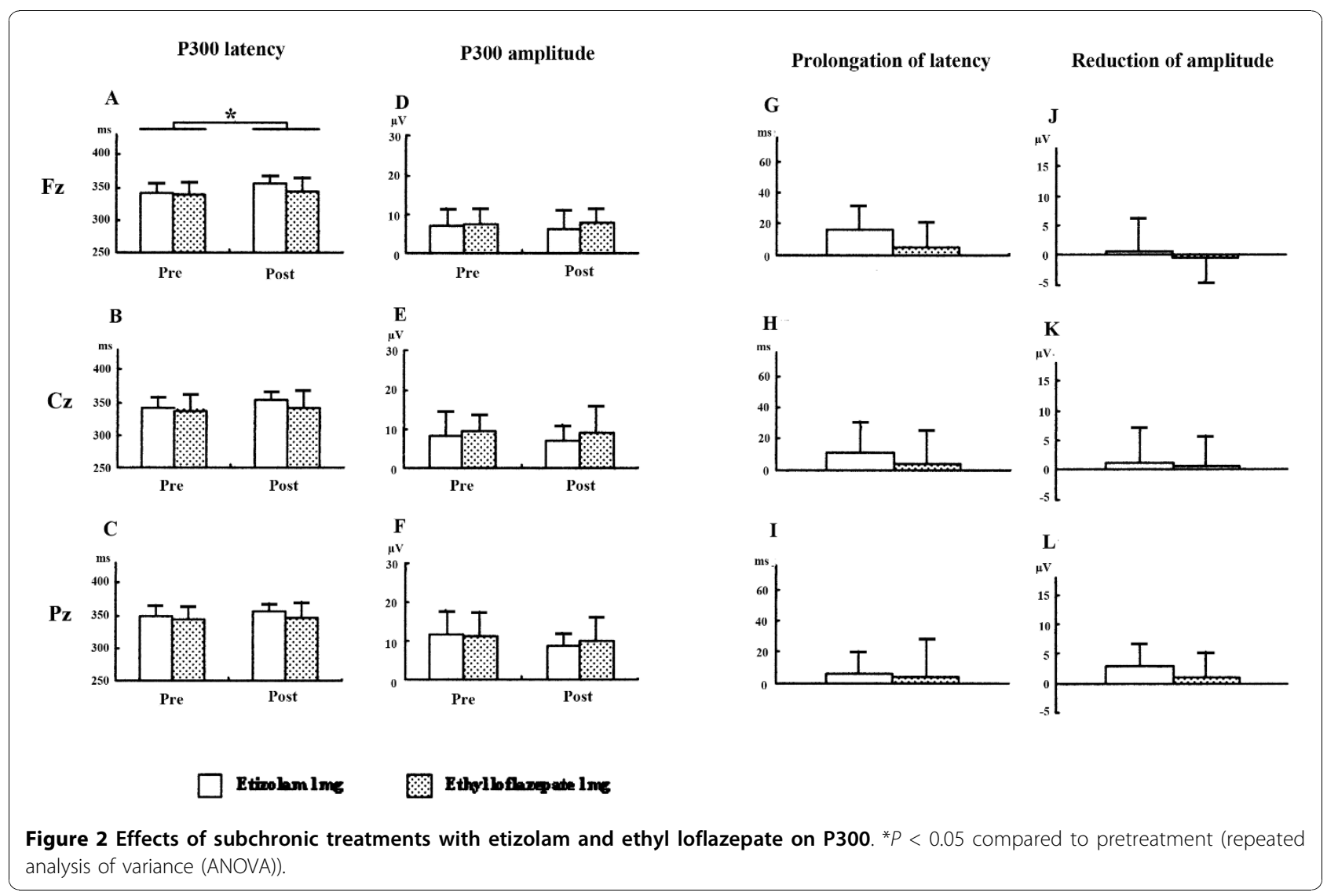




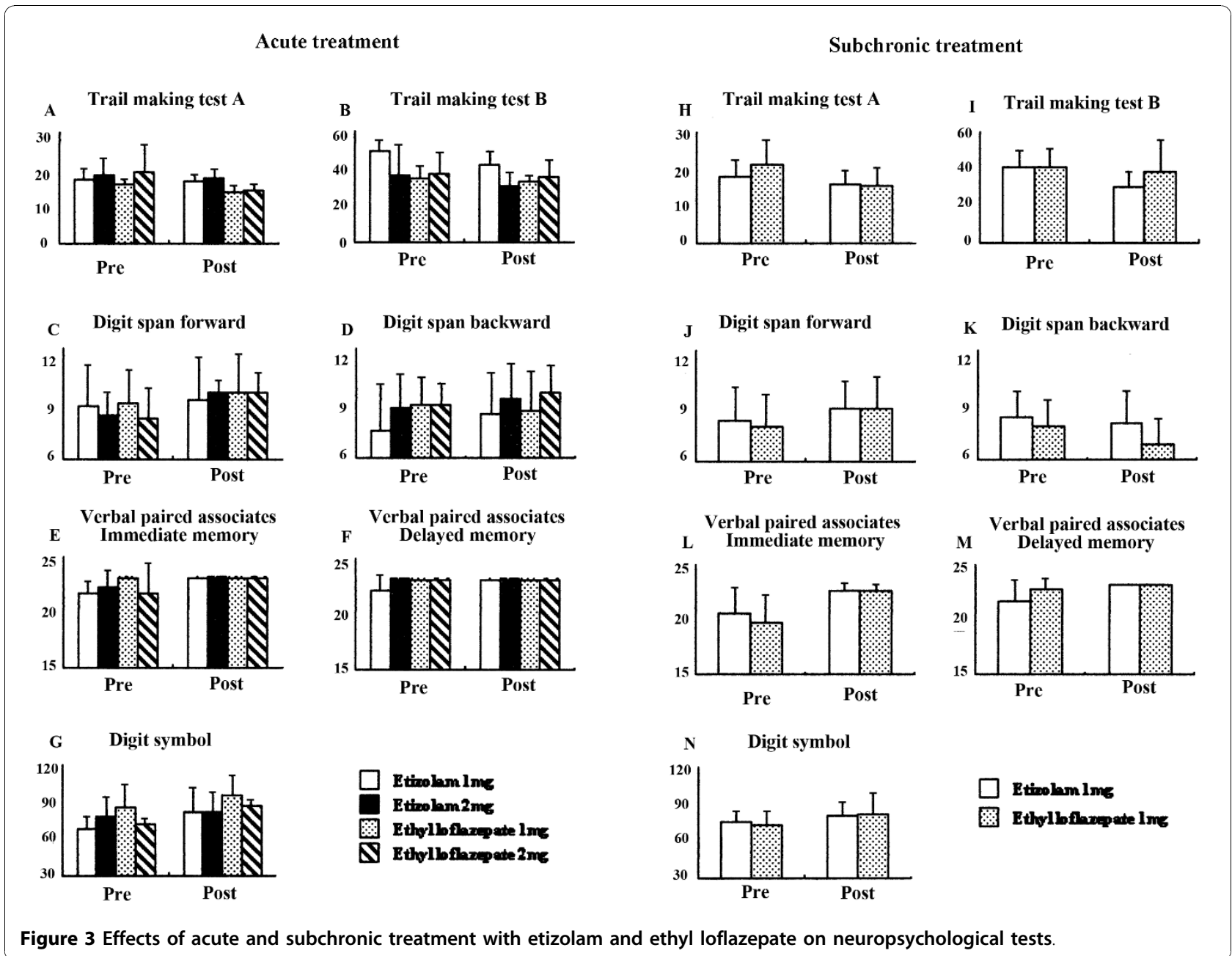

between the acute effects of etizolam and ethyl loflazepate could contribute to the potent sedative effects of etizolam, although equivalent doses of these two drugs to diazepam are clinically almost the same. P300 latency is suggested to reflect the stimulus evaluation time, and is relatively independent of response selection and execution [28-30]. Therefore, it is conceivable that ethyl loflazepate has less effect on P300-related information processing, although the subjects did not exhibit any harmful effects on motor skills, visuomotor tracking speed, and delayed memory in the neuropsychological tests.

Secondly, subchronic treatment with drugs produced prolongation in P300 latency only in the Fz regions. Weak prolongation in P300 latency was seen in the etizolam-treated subjects (although this was not statistically significant). The magnitude of prolongation by subchronic ethizolam treatment was reduced when compared to the acute administration of etizolam. In support of this finding, previous studies reported that people develop tolerance to the sedative and cognitive effects of benzodiazepines after subchronic treatments [1]. Interestingly, subchronic treatment of ethyl loflazepate did not prolong the latency in spite of its long elimination rate.

Finally, acute but not subchronic treatment with benzodiazepine reduced P300 amplitude. Based on the magnitude of changes, the main effects on the reduction of P300 amplitude were produced by etizolam $(2 \mathrm{mg})$. This result replicated previous studies that benzodiazepine anxiolytic drugs (lorazepam, clonazepam and alprazolam) induced reductions in P300 amplitude [15,17]. Recent studies demonstrated that reduction in auditory P300 amplitude correlated with the severity of thought disorders [31,32]. Previous studies reported that a single administration of a benzodiazepine drug produced impairment of learning and memory [1-3]. However, the present study showed no aversive effects of the examined drugs on neuropsychological tasks such as attention-needed tasks (trails making test, digit span) and memory (verbal paired associates, digit symbol). Since the subjects were free from abnormal pathological 
process, alterations in the P300 may be induced by etizolam, not by symptom alleviation due to etizolam.

Differences in the effects on P300 latency between etizolam and ethyl loflazepate could be attributed to their pharmacological properties, such as sedative effects, and affinities for $\omega-1$ and $\omega-2$ sites. Etizolam is short acting $(\mathrm{EH}$ of $6 \mathrm{~h})$ whereas ethyl loflazepate is long acting $(\mathrm{EH}$ of $122 \mathrm{~h}$ ).

With regard to limitations of the present study, the sample size was small.

\section{Conclusions}

Acute administration of etizolam induced significant prolongation in P300 latency whereas low dose ethyl loflazepate induced fewer effects on P300 latency in the $\mathrm{Fz}, \mathrm{Cz}$ and $\mathrm{Pz}$ regions than low-dose etizolam. For a while, subchronic administration of etizolam, but not ethyl loflazepate, caused weak prolongation in P300 latency in the $\mathrm{Fz}$ but not $\mathrm{Cz}$ and $\mathrm{Pz}$ regions. In contrast, acute and chronic administrations of etizolam and ethyl loflazepate showed no deficits in motor skills, visuomotor tracking speed, and delayed memory on neuropsychological testing.

\section{Author details}

'Department of Psychiatry, Chiba University Graduate School of Medicine, Chiba, Japan. ${ }^{2}$ Division of Clinical Neuroscience, Chiba University Center for Forensic Mental Health, Chiba, Japan.

\section{Authors' contributions}

GF conceived the paper, designed the study, performed the psychological measures, collected data, carried out the statistical analysis and drafted the paper; THash performed the psychological measures; YS carried out the statistical analysis and helped draft the study; THase, HW and MF supervised the study; $\mathrm{KH}$ and $\mathrm{Ml}$ designed the study and helped draft the papers. All authors read and approved the final manuscript.

\section{Competing interests}

The authors declare that they have no competing interests.

Received: 13 June 2010 Accepted: 3 November 2010

Published: 3 November 2010

\section{References}

1. Files SE, Lister RG: Lorazepam-induced deficits in learning result from impaired rehearsal, reduced motivation, or increased sedation? $\mathrm{Br} J \mathrm{Clin}$ Pharmacol 1982, 14:545-550.

2. Block Rl, Berchou R: Alprazolam and lorazepam effects on memory acquisition and retrieval processes. Pharmac Biochem Behav 1984, 20:233-241.

3. Curran HV: Tranquilizing memories: a review of the effects of benzodiazepines on human memory. Biol Psychology 1986, 23:179-213.

4. Chouinard GJ: Issues in the clinical use of benzodiazepines: potency, withdrawal, and rebound. J Clin Psychiatry 2004, 65(Suppl 5):7-12.

5. Stewart SA: The effects of benzodiazepines on cognition. J Clin Psychiatry 2005, 66(Suppl 2):9-13.

6. Urata J, Uchiyama M, lyo M, Enomoto T, Hayakawa T, Tomiyama M, Nkajima T, Sasaki H, Shirakawa S, Wada K, Fukui S, Yamadera H, Okawa M: Effects of a small dose of triazolam on P300 and resting EEG. Psychopharmacology 1996, 125:179-184.

7. Polich J, Kok A: Cognitive and biological determinants of P300: an integrative review. Biol Psychology 1995, 41:103-146.
8. Emmerson RY, Dustman RE, Shearer DE, Turner CW: P3 latency and symbol digit performance correlations in aging. Exp Aging Res 1989, 15:151-159.

9. O'Donell BF, Friedman S, Swearer JM, Drachman DA: Active and passive P3 latency and psychometric performance: influence of age and individual differences. Int J Psychophysiol 1992, 12:187-195.

10. Knight RT, Scabini D: Anatomic bases of event-related potentials and their relationship to novelty detection in humans. J Clin Neurophysiol 1998, 15:3-13.

11. Fjell AM, Walhovd KB: P300 and neuropsychological tests as measures of aging: salp topography and cognitive changes. Brain Topogr 2001, 14:25-40.

12. McAllister-Williams RH, Massey AE, Rugg MD: Effects of tryptophan depletion on brain potential correlates of episodic memory retrieval. Psychopharmacology 2002, 160:434-442.

13. Walhoved KB, Fjell AM: The relationship between P3 and neuropsychological function in an adult life span sample. Biol Psychology 2003, 62:65-87.

14. Pogarell O, Mulert C, Hegerl U: Event related potentials and fMRI in neuropsychopharmacology. Clin EEG Neurosci 2006, 37:99-107.

15. Berchou R, Chiyasirisobhon $S$, Green $V$, Mason K: The pharmacodynamic properties of lorazepam and methylphenidate drugs on event-related potentials and power spectral analysis in normal subjects. Clin Electroencephalogr 1986, 17:176-180.

16. Shinotoh $H$, lyo $M$, Yamada $T$, Inoue $O$, Suzuki $K$, Itoh $T$, Fukuda $H$, Yamasaki T, Tateno $Y$, Hirayama K: Detection of benzodiazepine receptor occupancy in the human brain by positron emission tomography. Psychopharmacology 1989, 99:202-207.

17. Rockstroh B, Elbert T, Lutzenberger W, Altenmuller E: Effects of the anticonvulsant benzodiazepine clonazepam on event-related brain potentials in humans. Electroencephalogr Clin Neurophysiol 1991, 78:142-149.

18. Semlitsch HV, Anderer $P$, Saletu B: Acute effects of the anxiolytics suriclone and alprazolam on cognitive information processing utilizing topographic mapping of event-related brain potentials (P300) in healthy subjects. Eur J Clin Pharmacol 1995, 49:183-191.

19. Nichols JM, Martin F: The effect of lorazepam on memory and eventrelated potentials in heavy and light social drinkers. Psychophysiology 1996, 33:446-456.

20. Hayakawa T, Uchikawa M, Urata J, Enomoto T, Okubo J, Okawa M: Effects of small dose of triazolam on P300. Psychiatry Clin Neurosci 1999, 53:185-187.

21. Chambon JP, Perio A, Demarne H, Hallot A, Dantzer R, Roncucci R, Biziere K: Ethyl loflazepate: a prodrug from the benzodiazepine series designed to dissociate anxiolytic and sedative activities. Arzneimittelforschung 1985, 35:1573-1577.

22. Tsumagari T, Nakajima A, Fukuda $T$, Shuto $S$, Kenjo $T$, Morimoto $Y$, Takigawa Y: Pharmacological properties of 6-(o-chlorophenyl)-8-ethyl-1methyl-4H-s-triazolo[3,4-c]thieno[2,3-e] [1,4]diazepine (Y-7131), a new anti-anxiety drug. Arzneimittelforschung 1978, 28:1158-64.

23. Inagaki A, Inada T, Fujii Y, Yagi K, Yoshio T, Nakamura H, Yamauchi T: Dose equivalence of psychotropic drugs [in Japanese] Tokyo, Japan: Seiwa Shoten Co. Ltd; 1999, 99-121.

24. Inada T, Nozaki S, Inagaki A, Furukawa TA: Efficacy of diazepam as an antianxiety agent: meta-analysis of double-blind, randomized controlled trials carried out in Japan. Human Psychopharmac Clin Exp 2003, 18:483-487.

25. Reitan RM, Wolfson D: The Halstead-Reitan Neuropsychological Test Battery Tucson, AZ, USA: Neuropsychology Press; 1985.

26. Smith A: Symbol Digit Modalities Test (SDMT): Manual (revised) Los Angeles, CA, USA: Western Psychological Services; 1982.

27. Wechsler DA: Wechsler Memory Scale-Revised, Manual New York, USA: Psychological Corp; 1987.

28. Kutas M, McCarthy G, Donchin E: Augmenting mental chronometry: the P300 as a measure of stimulus evaluation time. Science 1977, 197:792-795.

29. McCarthy G, Donchin E: A metric for thought: a comparison of P300 latency and reaction time. Science 1981, 211:77-80.

30. Magliero A, Bashoe TR, Coles MG, Donchin E: On the dependence of P300 latency on stimulus evaluation processes. Psychophysiology 1984, 21:171-186. 
31. Higashima M, Nagasawa T, Kawasaki Y, Oka T, Sakai N, Tsukada T, Koshino Y: Auditory P300 amplitude as a state marker for positive symptoms in schizophrenia: cross-sectional and retrospective longitudinal studies. Schizophr Res 2001, 59:147-157.

32. Iwanami A, Okajima Y, Isono H, Shinoda J, Kasai K, Hata A, Fukuda M, Nakagome K, Kamijima K: Effects of risperidone on event-related potential in schizophrenic patients. Pharmacopsychiatry 2001, 34:73-79.

doi:10.1186/1744-859X-9-37

Cite this article as: Fukami et al.: Effects of etizolam and ethyl

loflazepate on the P300 event-related potential in healthy subjects. Annals of General Psychiatry 2010 9:37.

Submit your next manuscript to BioMed Central and take full advantage of:

- Convenient online submission

- Thorough peer review

- No space constraints or color figure charges

- Immediate publication on acceptance

- Inclusion in PubMed, CAS, Scopus and Google Scholar

- Research which is freely available for redistribution

Submit your manuscript at www.biomedcentral.com/submit 